\title{
ANÁlise TermodinâmiCA de Motores de CoMbustão InTERna de TIPO ALTERNATIVO USANDO UM MODELO QUASI-DIMENSIONAL
}

\author{
Daniel da Silva Tonon, Ezio Castejon Garcia \\ Instituto Tecnológico de Aeronáutica (ITA) - Divisão de Engenharia Mecânica \\ E-mails: ds.tonon@gmail.com, ezio@ita.br
}

\section{RESUMO}

O uso de simulações para a predição do comportamento de determinados mecanismos está cada vez mais difundido na indústria automobilística, uma vez que ensaios experimentais possuem um custo muito mais elevado. Dentre todos os mecanismos que compõem um automóvel o motor merece um destaque especial, já que este é responsável pela geração de potência e é através dele que o veículo iniciará o seu movimento. Com o passar do tempo o projeto desse mecanismo precisou evoluir consideravelmente se comparado com os primeiros automóveis fabricados na história, devido as exigências de âmbito social e ecológico. Dessa forma, é de fundamental importância que as montadoras avaliem o comportamento do motor em toda a sua faixa de operação, e essa avaliação pode ser feita através de diferentes modelos numéricos. Atualmente os modelos mais difundidos para a simulação desses mecanismos são modelos Single-Zone, Multi-Zone e Multi-Dimensional. O presente trabalho faz uma análise comparativa entre dados experimentais e resultados obtidos através de simulações de motores de combustão interna, utilizando um modelo Multi-Zone de tipo Quasi-Dimensional. O modelo apresentado nesse trabalho ainda está em fase de desenvolvimento, porém já demonstra um grande potencial nas análises de desempenho.

\section{NOMENCLATURA}

T: temperatura

$T_{u}$ : temperatura dos gases não queimados

$T_{b}$ : temperatura dos gases queimados

$T_{\text {ref: }}$ temperatura de referência $(297 \mathrm{~K})$

$T_{\text {wall }}$ : temperatura da parede

$T_{r}$ : temperatura após a admissão

$m_{i n}:$ massa entrando

$m_{e x}$ : massa saindo

$m_{u}$ : massa não queimada

$m_{b}$ : massa queimada

$c_{p}$ : calor específico a pressão constante

$c_{p u}$ : calor específico a pressão constante da massa não queimada

$c_{p b}$ : calor específico a pressão constante da massa queimada

$Q$ : calor transferido

$Q_{u}$ : calor transferido pela massa não queimada $\rho_{b}$ : densidade da massa queimada
$Q_{b}$ : calor transferido pela massa queimada

$h_{i n}$ : entalpia da massa entrando

$h_{e x}$ : entalpia da massa saindo

$h_{u}$ : entalpia da massa não queimada

$h_{b}$ : entalpia da massa queimada

$V$ : volume total

$V_{u}$ : volume não queimado

$V_{b}$ : volume queimado

$V_{r}$ : pressão após a admissão

$V_{d t}$ : volume varrido

$p$ : pressão

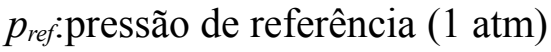

$p_{r}$ : pressão após a admissão

$p_{m}$ : pressão em vazio

$\rho$ : densidade

$\rho_{u}$ : densidade da massa não queimada 
$R_{u}$ : constante independente do gás para a massa não queimada

$R_{b}$ : constante independente do gás para a massa queimada

$S_{L}$ : velocidade de propagação de frente de chama laminar

$S_{L, 0}$ : velocidade de propagação de frente de chama laminar de referência

$S_{T}$ : velocidade de propagação de frente de chama turbulenta

$f$ : fator fenomenológico

$x_{b}$ : fração de massa queimada

rot: velocidade em rotações por minuto

$y_{r}$ : fração de massa queimada residual

$\alpha$ : coeficiente exponencial

$\beta$ : coeficiente exponencial

$\phi$ : razão de equivalência $\phi_{m}$ : parâmetro de razão de equivalência para $S_{L, 0}$ máximo

$B_{m}$ : parâmetro para cálculo de $S_{L, 0}$

$B_{\phi}$ : parâmetro para cálculo de $S_{L, 0}$

$n$ : número de mols de uma dada espécie

$N$ : número de mols totais

$a_{s}$ : número de mols de ar para combustão estequiométrica

$y$ : fração molar

$K$ : constante de equilíbrio

$h_{\text {wos }}$ : coeficiente de transferência de calor

$u$ : velocidade característica

$k$ : coeficiente politrópico

$D$ : diâmetro do cilindro

$v_{p}$ : velocidade média do pistão

: representação da taxa de uma propriedade ou variável com o tempo

\section{INTRODUÇÃO}

A simulação de motores de combustão interna, atualmente, é feita através de três tipos de modelos, sendo eles: Single-Zone, Multi-Zone e Multi-Dimensional. Com relação ao primeiro, a câmara de combustão é analisada como uma única região, de forma que todas as propriedades são homogêneas. Os modelos do segundo tipo, Multi-Zone, dividem a câmara de combustão em várias regiões, que podem ter propriedades distintas umas das outras, por exemplo, pode haver diferença de temperatura entre uma região e outra. Por fim, os modelos Multi-Dimensional também dividem a análise em várias seções, porém essas possuem mais de uma dimensão física.

A motivação inicial é desenvolver um código próprio e simplificado, capaz de analisar o comportamento de motores de combustão interna de ciclo Otto, porém deseja-se que no futuro esse código seja atualizado, de forma a conseguir estimar parâmetros de desempenho, consumo e emissões. Dessa forma, optou-se por utilizar um modelo de tipo Multi-Zone, já que este permite analisar efeitos de estratificação de temperatura e espécies químicas, o que possibilitará uma melhor estimativa de parâmetros como a duração da combustão, a evolução da pressão durante a combustão, e as emissões[1].

O equacionamento utilizado para a construção do código é descrito por Curto[1], que propõe um modelo Multi-Zone de tipo Quasi-Dimensional para a análise desses mecanismos.

É importante ressaltar que o modelo proposto neste trabalho ainda está em fase de desenvolvimento, e todas as análises relatadas neste texto são de cunho qualitativo, e não quantitativo, de forma que o objetivo principal é conseguir reproduzir o comportamento dos dados utilizados.

\section{MAIS DO QUE UMA QUESTÃO DE NOMENCLATURA}

O ciclo motor é composto de 6 eventos: admissão, compressão, combustão, expansão motora, blowdown e exaustão. Na língua inglesa a classificação dos motores de combustão interna é 
feita através do número de vezes que o pistão percorre a distância entre o Ponto Morto Inferior (PMI) e o Ponto Morto Superior (PMS), até que todos esses eventos sejam realizados. Dessa forma, os motores podem ser de dois tipos: 2 stroke e 4 stroke (stroke é a palavra utilizada na língua inglesa para definir a distância entre o PMI e o PMS).

Essa mesma classificação no Brasil é feita utilizando a palavra "tempos" (motores de 2 ou 4 "tempos"), porém, na língua portuguesa, a distância entre o PMI e o PMS é definida como curso. Neste trabalho faz-se uma substituição da palavra "tempos" para "cursos", de forma a classificar os motores de combustão interna como sendo de 2 ou 4 cursos.

\section{MODELOS MULTI-ZONE}

Antes de se analisar um modelo Multi-Zone, deve-se entender como trabalham os modelos Single-Zone nas simulações de motores de combustão interna.

Modelos Single-Zone são modelos numéricos onde a única variável independente é o tempo [1]. Dessa forma esses modelos consideram que a composição e as propriedades termodinâmicas, como temperatura e pressão, são espacialmente uniforme, e variarão apenas pela influência do tempo [2]. Esses tipos de modelos geralmente utilizam uma relação empírica para determinar a taxa de consumo de combustível, ou funções analíticas, como, por exemplo, a curva de Wiebe [3].

No caso dos modelos Multi-Zone, a câmara de combustão é dividida em várias regiões. Cada uma dessas regiões poderá trocar calor e massa com as regiões adjacentes, seja essa uma outra região da câmara de combustão ou a parede do cilindro. A temperatura de cada uma dessas regiões é distinta e homogênea dentro daquela região, e naturalmente a pressão para todas as regiões é igual. Figura 1 mostra um exemplo de divisão da câmara de combustão em um modelo Multi-Zone.

Figura 1: Exemplo da divisão da câmara de combustão para um modelo Multi-Zone com $n$ regiões.

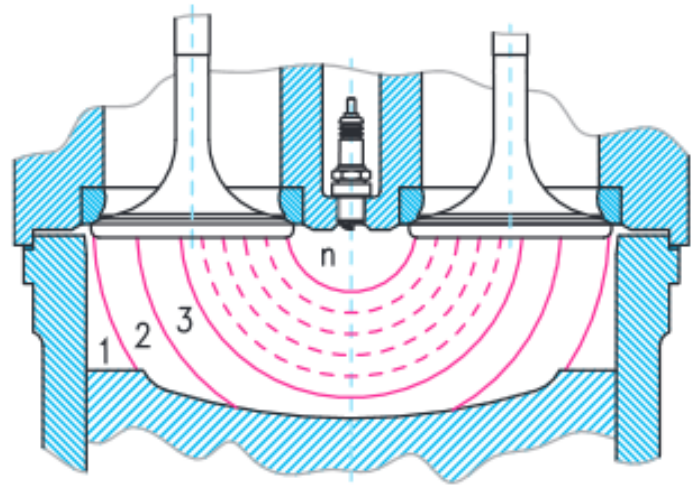

Fonte: Hradec (2012) [4].

A grande vantagem desses modelos em relação aos modelos Single-Zone é o fato de se poder verificar fenômenos mais complexos, como a estratificação de temperatura, ou de espécies dentro da câmara de combustão, e esses tipos de informação são importantes para estimar a duração da combustão, e a taxa de aumento da pressão com o andamento do ciclo.

No caso desse texto, porém, utiliza-se um caso particular dos modelos Multi-Zone, que são os modelos Quasi-Dimensional. Estes dividem a câmara de combustão em apenas duas regiões, 
e apenas durante o evento de combustão [1]. Assume-se que durante a combustão a chama se propagará como uma calota esférica, preenchendo o volume da câmara de combustão até que não exista mais espécies a serem oxidadas na câmara. A forma de se determinar a evolução dessa calota esférica pode ser feita utilizando equações analíticas, como a curva de Wiebe, ou com o auxílio de um submodelo que determine a velocidade de propagação de frente de chama [3]. O modelo proposto nesse trabalho utiliza um submodelo para o cálculo da velocidade de propagação de frente de chama, que será discutido em outra parte do texto.

Outros tipos de modelo que podem ser citados como alternativa para a simulação de Motores de Combustão Interna, são os modelos Multi-Dimensional, que estão fortemente associados a análises CFD, porém estes não são muito utilizados para a determinação de parâmetros como torque e potência, pois exigem um tempo de cálculo muito mais elevado que os modelos anteriormente descritos $[1,5]$. Essas análises são mais utilizadas em casos específicos, como a determinação da temperatura da sede da válvula de exaustão, por exemplo.

\subsection{Equacionamento}

O desenvolvimento do equacionamento não será demonstrado nesse documento, mas pode ser encontrado em [1]. Aqui apenas serão mostradas as principais equações utilizadas para a obtenção da Temperatura e Pressão dentro da câmara de combustão.

A taxa de variação da temperatura com o tempo, é obtida através da seguinte equação:

$\dot{T}=\frac{1}{\left(m_{u} \cdot c_{p, u}+m_{b} \cdot c_{p . b}\right)} \cdot\left[\dot{Q}_{u}+\dot{Q}_{b}+\dot{m}_{\mathrm{in}} \cdot h_{\mathrm{in}}-\dot{m}_{u} \cdot h_{u}+\dot{m}_{\mathrm{ex}} \cdot h_{\mathrm{ex}}-\dot{m}_{b} \cdot h_{b}+V \cdot \dot{p}\right]$

Para a taxa de variação da pressão com o tempo:

$\dot{p}=\left[p \cdot\left(\frac{\dot{m}_{u}}{\rho_{u}}+\frac{\dot{m}_{b}}{\rho_{b}}-\dot{V}\right)+\zeta \cdot\left(\dot{Q}_{u}+\dot{Q}_{b}+\dot{m}_{\mathrm{in}} \cdot h_{\mathrm{in}}-\dot{m}_{u} \cdot h_{u}+\dot{m}_{\mathrm{ex}} \cdot h_{\mathrm{ex}}-\dot{m}_{b} \cdot h_{b}\right)\right] \cdot \frac{1}{[V \cdot(1-\zeta)]}$

Sendo que:

$\zeta=\frac{V}{\frac{V_{u} \cdot c_{p, u}}{R_{u}}+\frac{V_{b} \cdot c_{p, b}}{R_{b}}}$

Evidentemente nem todos os eventos do ciclo motor necessitarão de todos esses parâmetros presentes nas equações (1), (2) e (3). Note que, durante o evento de admissão a válvula de exaustão estará fechada, e dessa forma não haverá massa saindo do volume de controle, e portanto esse termo será igual a zero. Além disso, ainda durante a admissão, a taxa de massa entrando no volume de controle será igual à taxa de massa não queimada no volume de controle. O mesmo tipo de raciocínio pode ser estendido para o evento de exaustão. Note também que durante a compressão e a expansão motora, a câmara de combustão será um sistema fechado, 
de forma que todos os termos referentes as entradas e saídas de massa serão iguais a zero.

\subsection{Submodelo de Combustão}

Para esse submodelo assume-se que assim que a vela é acionada a combustão se inicia no ponto onde a centelha é gerada, e assim a frente de chama se propagará por toda a câmara de combustão com o formato de uma calota esférica. Figura 2 representa esse tipo de comportamento.

Figura 2: Representação da propagação da chama pela câmara de combustão.

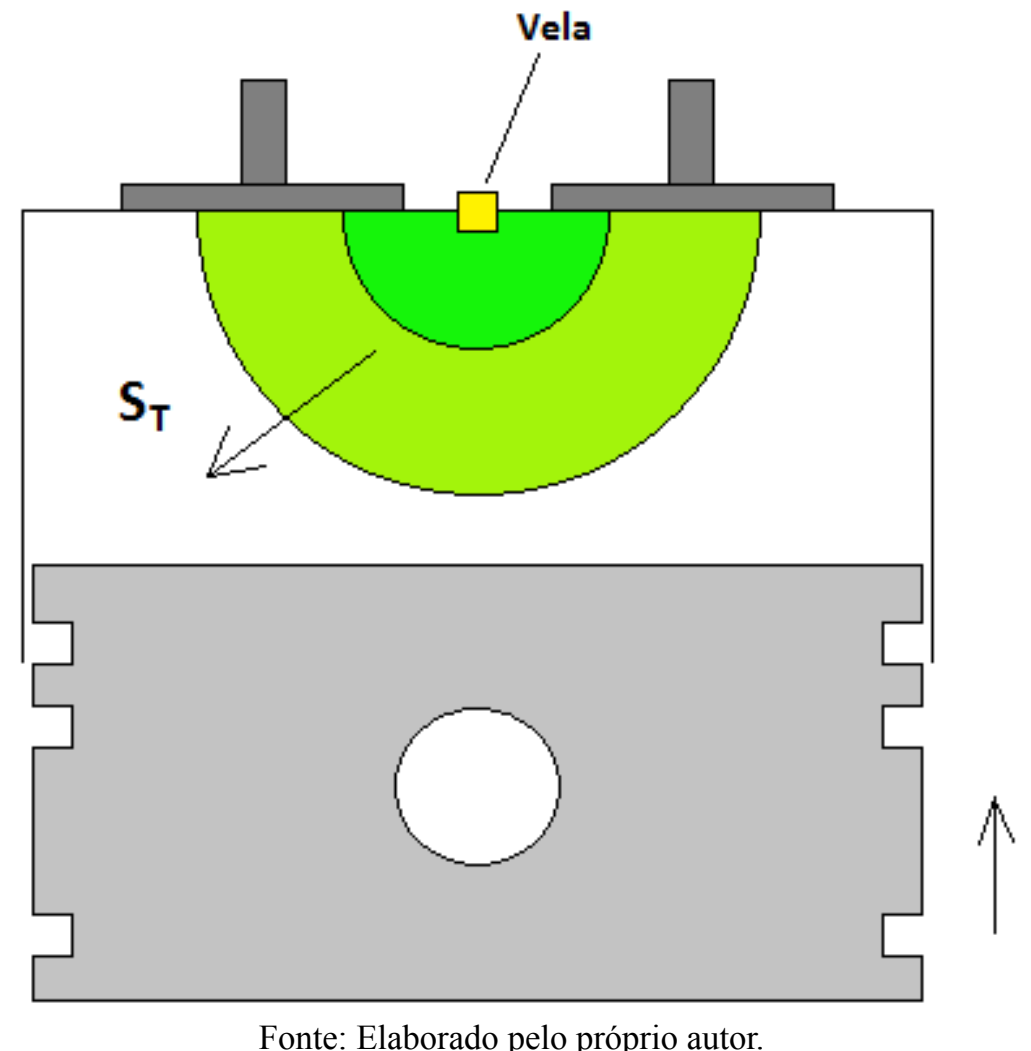

Assim como mostrado na Figura 2, a chama se propaga com uma intensidade igual à velocidade de propagação de frente de chama turbulenta, referente ao combustível e às condições termodinâmicas dentro da câmara de combustão, para cada instante.

A seguinte correlação [1], propõem o cálculo da velocidade de propagação de frente de chama turbulenta de uma forma proporcional à velocidade de propagação de frente de chama laminar:

$$
S_{T}=f \cdot S_{L} \cdot \frac{\frac{\rho_{u}}{\rho_{b}}}{\left(\frac{\rho_{u}}{\rho_{b}}-1\right) \cdot x_{b}+1}
$$


O fator fenomenológico, $f$ [1], presente na equação (4), é calculado da seguinte forma:

$f=1+0,0018 \cdot r o t$

Para o cálculo da velocidade de propagação de chama laminar, utiliza-se a correlação de Kerk, descrita em Heywood [3]:

$S_{L}=S_{L, 0} \cdot\left(\frac{T_{u}}{T_{r e f}}\right)^{\alpha} \cdot\left(\frac{p}{p_{\text {ref }}}\right)^{\beta} \cdot\left(1-2,06 \cdot y_{r}^{0,77}\right)$

Os coeficientes $\alpha, \beta$ e $S_{L, 0}$, são obtidos da seguinte forma:

$$
\begin{aligned}
& \alpha=2,18-0,8 \cdot(\phi-1) \\
& \beta=-0,16+0,22 \cdot(\phi-1) \\
& S_{L, 0}=B_{m}+B_{\phi} \cdot\left(\phi-\phi_{m}\right)^{2}
\end{aligned}
$$

Os valores de $\phi_{m}, B_{m}$ e $B_{\phi}$, são referentes ao combustível, que no caso desse trabalho foi considerado o isoctano. Esses valores podem ser encontrados em [3].

Note que as equações apresentadas não são capazes de considerar alguns fatores importantes e que exercerão forte influência na propagação da chama na câmara de combustão, como por exemplo a modificação do raio referente a frente de chama, devido a compressão ou expansão com o movimento do pistão, e os efeitos que a parede da câmara de combustão exercerá quando a frente de chama encontra esse obstáculo. Dessa forma inseriu-se no modelo um fator de correção $F$ multiplicando a equação (4), de forma a ajustar o modelo. Esse fator foi inserido devido a limitação do código em prever o instante exato de início de liberação de energia [3], além dos efeitos de turbulência e influência das paredes [1], na combustão. Esse fator é dado da seguinte forma:

$$
\begin{array}{lll}
F=\operatorname{sen}\left(\frac{0,5 \cdot \pi}{3}+\frac{\pi}{3} \cdot \frac{x_{b}}{0,25}\right) & \rightarrow & 0<x_{b}<0,25 \\
F=1 & \rightarrow \quad 0,25<x_{b}<0,65 \\
F=\operatorname{sen}\left(\frac{\pi}{2}-\frac{\pi}{3} \cdot\left(\frac{\left(x_{b}-0,65\right)}{0,35}\right)\right) & \rightarrow & 0,65<x_{b}<1
\end{array}
$$

\subsection{Reações Químicas}

Durante a combustão a mistura de ar mais combustível passará por um processo de oxidação, de forma que essa mistura será convertida em outras espécies, que serão chamadas de produtos de combustão. O número de espécies e de reações intermediárias durante esse processo pode ser gigantesco, se tornando inviável para as análises propostas pelo modelo apresentado.

Dessa forma, adota-se um modelo de reações químicas mais simples, porém suficiente para as análises desejadas. Esse modelo é descrido em Ferguson [6], e 
considera a seguinte reação global, para um dado combustível $\mathrm{C}_{\alpha} \mathrm{H}_{\beta} \mathrm{O}_{\gamma} \mathrm{N}_{\delta}$, com proporção estequiométrica $a_{s}$, e relação de equivalência $\phi$ :

$$
\begin{aligned}
C_{\alpha} H_{\beta} O_{\gamma} N_{\delta}+\frac{a_{s}}{\phi} \cdot\left(O_{2}+3,76 N_{2}\right) \rightarrow & n_{1} \cdot \mathrm{CO}_{2}+n_{2} \cdot H_{2} \mathrm{O}+n_{3} \cdot N_{2}+n_{4} \cdot \mathrm{O}_{2}+n_{5} \cdot \mathrm{CO}+ \\
& \mathrm{n}_{6} \cdot \mathrm{H}_{2}+n_{7} \cdot H+n_{8} \cdot \mathrm{O}+n_{9} \cdot \mathrm{O}+n_{9} \cdot \mathrm{OH}+n_{10} \cdot \mathrm{NO}
\end{aligned}
$$

Deve-se, portanto, encontrar o número de mols de cada espécie dos produtos de combustão. Através do balanço químico dos componentes, tem-se:

$$
\begin{aligned}
& C: \quad \alpha=\left(y_{1}+y_{5}\right) \cdot N \\
& H: \quad \beta=\left(2 \cdot y_{2}+2 \cdot y_{6}+y_{7}+y_{9}\right) \cdot N \\
& O: \quad \gamma+\frac{2 \cdot a_{s}}{\phi}=\left(2 \cdot y_{1}+y_{2}+2 \cdot y_{4}+y_{5}+y_{8}+y_{9}+y_{10}\right) \cdot N \\
& N: \quad \delta+\frac{3,76 \cdot a_{s}}{\phi}=\left(2 \cdot y_{3}+y_{10}\right) \cdot N
\end{aligned}
$$

Sabe-se também que:

$$
\left(\sum_{i=1}^{10} y_{i}\right)-1=0
$$

Contudo, nota-se que existem 11 incógnitas (as frações molares de cada componente e mais o número de mols total), e apenas 5 equações. As outras seis equações são dadas das constantes de equilíbrio de algumas reações, sendo elas:

$$
\begin{array}{lrl}
\frac{1}{2} \cdot H_{2} \Leftrightarrow H & K_{1}=\frac{y_{7} \cdot p^{0,5}}{y_{6}^{0,5}} \\
\frac{1}{2} \cdot O_{2} \Leftrightarrow O & K_{2}=\frac{y_{8} \cdot p^{0,5}}{y_{4}^{0,5}} \\
\frac{1}{2} \cdot O_{2}+\frac{1}{2} \cdot H_{2} \Leftrightarrow O H & K_{3}=\frac{y_{9}}{y_{4}^{0,5} \cdot y_{6}^{0,5}} \\
\frac{1}{2} \cdot O_{2}+\frac{1}{2} \cdot N_{2} \Leftrightarrow N O & K_{4}=\frac{y_{10}}{y_{4}^{0,5} \cdot y_{3}^{0,5}} \\
\frac{1}{2} \cdot O_{2}+H_{2} \Leftrightarrow H_{2} O & K_{5}=\frac{y_{2}}{y_{4}^{0,5} \cdot y_{6} \cdot p^{0,5}} \\
\frac{1}{2} \cdot O_{2}+C O \Leftrightarrow C O_{2} & K_{6}=\frac{y_{1}}{y_{4}^{0,5} \cdot y_{5} \cdot p^{0,5}}
\end{array}
$$

O valor de cada uma dessas constantes é obtido através da seguinte correlação:

$$
\log _{10} K_{i}(T)=A_{i} \cdot \ln \left(\frac{T}{1000}\right)+\frac{B_{i}}{T}+C_{i}+D_{i} \cdot T+E_{i} \cdot T^{2}
$$


Os valores de $A_{i}, B_{i}, C_{i}, D_{i}$ e $E_{i}$ para cada uma das constantes, é fornecido em [6].

\subsection{Trocas térmicas}

De maneira geral para a estimativa das trocas de calor, dois modelos são mais difundidos, sendo eles os modelos propostos por Annand (1970-1971) [7] e Woschni (1967) [8].

O primeiro tenta caracterizar as trocas de calor de maneira geral, ou seja, incluindo todos os mecanismos de troca de calor durante o ciclo. O segundo modelo (Woschni), que também é muito utilizado, diferentemente do modelo anterior, não considera as perdas por radiação como parte da equação, mas adiciona a contribuição deste mecanismo indiretamente.

Para esse trabalho escolheu-se trabalhar com o modelo de Woschni, que se baseia na seguinte equação:

$$
\dot{q}=h_{\text {wos }} \cdot\left(T-T_{\text {wall }}\right)
$$

Sendo que o coeficiente global de transferência de calor é obtido através da seguinte correlação:

$$
h_{w o s}=129,8 \cdot p^{0,8} \cdot D^{-0,2} \cdot T^{-0,53} \cdot u^{0,8}
$$

A velocidade característica é calculada da seguinte forma:

$$
u(t)=C_{1} \cdot v_{p}+C_{2} \cdot \frac{V_{d t} \cdot T_{r}}{p_{r} \cdot V_{r}} \cdot\left[p(t)-p_{m}\right]
$$

Sendo que a $p_{r}, T_{r}$ e $V_{r}$ são os valores dessas propriedades no momento em que a válvula de admissão é fechada. Os coeficientes $C_{1}$ e $C_{2}$ possuem valores diferentes para cada evento do ciclo motor e esses valores podem ser consultados em [1]. A pressão em vazio, $p_{m}$, pode ser calculada por uma relação politrópica, por exemplo:

$p_{m}=p_{r} \cdot\left(\frac{V_{r}}{V}\right)^{k}$

O valor do coeficiente politrópico pode variar de 1,2 até 1,4, dependendo do combustível.

\subsection{Trocas gasosas}

As trocas gasosas em um modelo do tipo apresentado nesse trabalho possui fundamental importância, pois aspectos como os pontos de abertura e fechamento das válvulas, overlap, lift, entre outros, são de fundamental importância para a estimativa de parâmetros de desempenho ou emissões. Porém, como já dito anteriormente, o modelo apresentado neste documento está ainda em fase primária de desenvolvimento, dessa forma nenhum desses aspectos do âmbito das trocas 
gasosas está sendo trabalhado, de forma que todas as aberturas e fechamentos das válvulas são considerados instantâneos.

\section{A LINGUAGEM DE PROGRAMAÇÃO PYTHON}

Python é uma linguagem de programação de alto nível que vem se difundido pelo mundo pela sua dinâmica e conteúdo disponível. Além de ser uma linguagem de programação de código aberto, e dessa forma a liberdade para a programação se torna muito maior. Além disso, conta com uma vasta quantidade de tutoriais e bibliotecas disponibilizadas, muitas vezes, pelos próprios usuários da linguagem.

Atualmente as principais aplicações desta linguagem estão voltadas para o desenvolvimento de sites, software e para aplicações científicas, apesar de existirem códigos sendo desenvolvidos para a execução de diversas outras funções que vão desde aplicativos de celular até aplicações caseiras. O modelo matemático completo deste trabalho é resolvido utilizando o pacote SciPy[9].

\section{RESULTADOS E DISCUSSÃO}

Nesta parte do texto serão apresentados alguns resultados obtidos com o modelo proposto. Deve-se lembrar que o objetivo não é alcançar elevada precisão dos resultados com o modelo em relação ou dados experimentais, deseja-se apenas representar o comportamento do fenômeno, de forma que o modelo possa ser futuramente melhorado. Todos os dados analisados são referentes a motores de 4 cursos.

Na figura 3, compara-se dados experimentais de um motor com 4 cilindros em linha, volume deslocado total de $1581 \mathrm{~cm}^{3}$, taxa de compressão igual a 9,2, diâmetro de $86,4 \mathrm{~mm}$, curso de $67,4 \mathrm{~mm}$, relação de equivalência 1,11 , velocidade de $3000 \mathrm{rpm}$ e em condição de plena carga. Estes dados experimentais foram obtidos de Millo [10]. Nota-se que os resultados obtidos com o modelo representam com grande fidelidade os dados experimentais, apresentando poucas discordâncias e reproduzindo o comportamento observado nos testes.

A figura 4 mostra os resultados obtidos da simulação de um motor com taxa de compressão 9,4, diâmetro de $72 \mathrm{~mm}$, curso de $61 \mathrm{~mm}$, relação de equivalência 1, velocidade de $1500 \mathrm{rpm}$ e em condição de carga mínima. Os dados experimentais foram obtidos de Chitragar(2015) [11]. Neste caso os resultados do modelo se distanciam dos experimentais, porém ainda notase que o comportamento é mantido, assim como o pico de pressão do ciclo.

A figura 5 mostra os resultados obtidos da simulação de um motor com taxa de compressão 10, diâmetro de $77,4 \mathrm{~mm}$, curso de $85 \mathrm{~mm}$, relação de equivalência 1 , ponto de ignição de 26 graus antes do PMS, velocidade de $1400 \mathrm{rpm}$ e uma pressão no coletor de admissão de 61,5 $\mathrm{kPa}$. Os dados experimentais foram obtidos de Ji(2016) [12]. Neste caso os resultados do modelo estão defasados dos dados experimentais, porém pode-se considerar que os resultados representam o comportamento observado na prática. 
Figura 3: Comparação dos dados de Millo [10] com os resultados simulados.

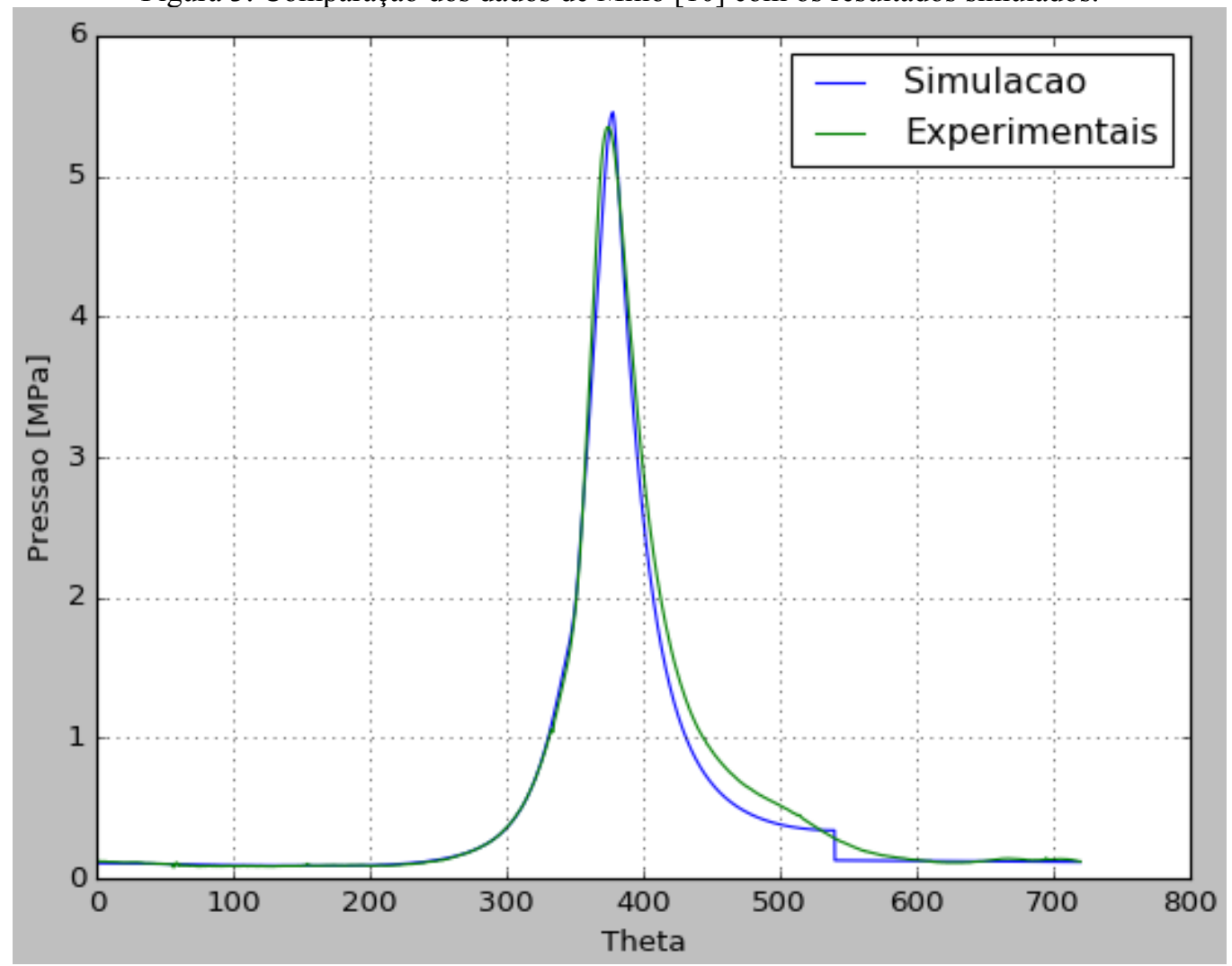

Fonte: Elaborado pelo próprio autor.

Figura 4: Comparação dos dados de Chitragar [11] com os resultados simulados.

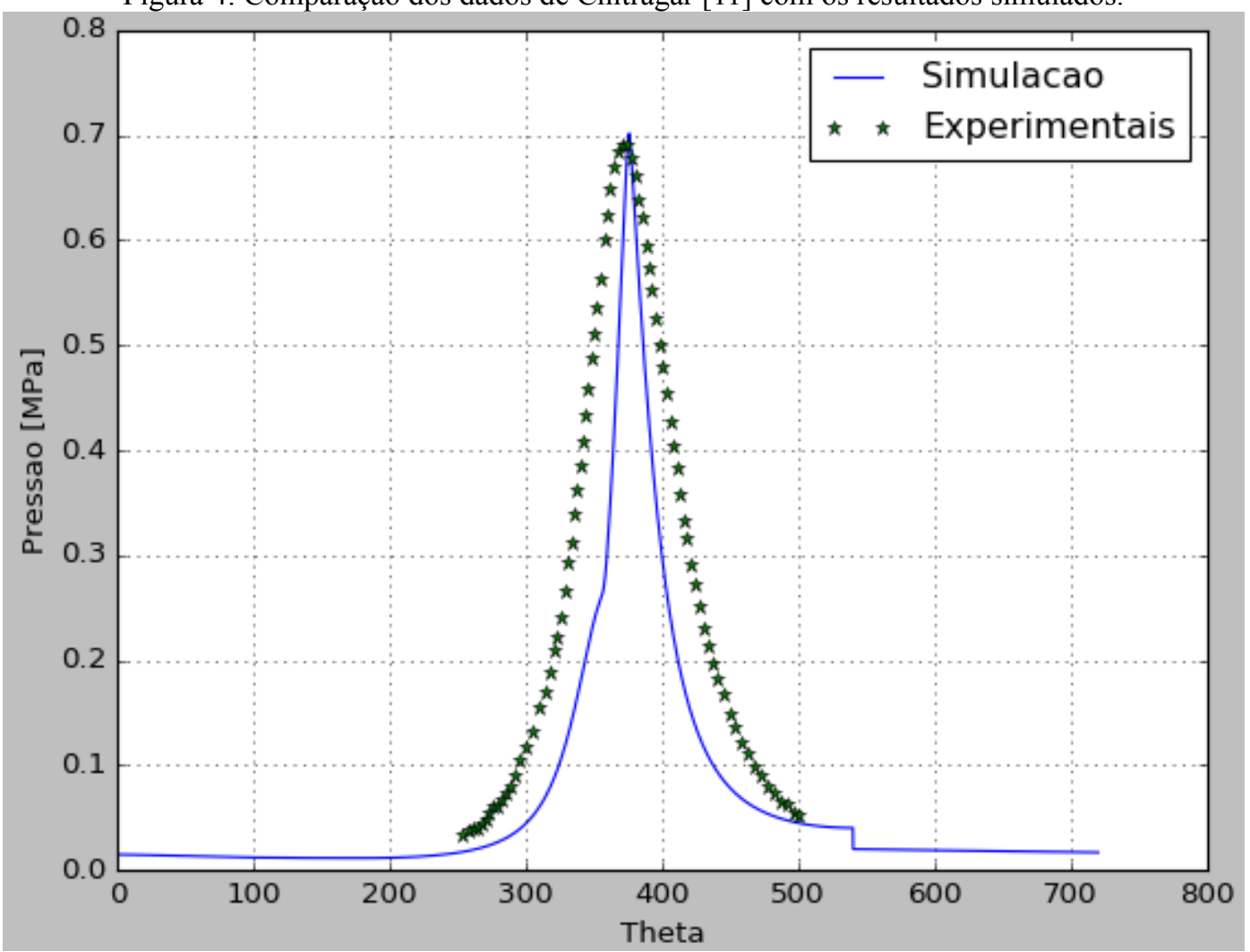

Fonte: Elaborado pelo próprio autor. 
Figura 5: Comparação dos dados de Ji [12] com os resultados simulados.

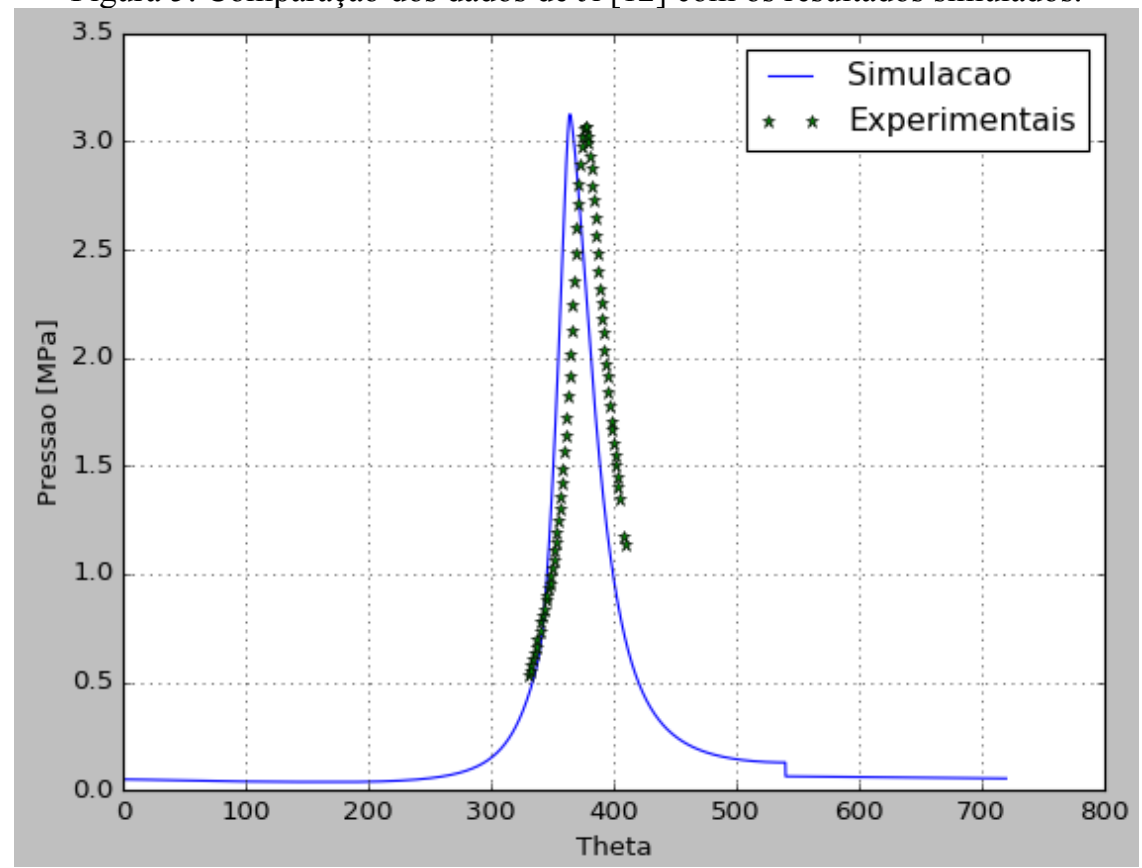

Fonte: Elaborado pelo próprio autor.

A figura 6 mostra os resultados obtidos da simulação de um motor com taxa de compressão 10, diâmetro de $77,4 \mathrm{~mm}$, curso de $85 \mathrm{~mm}$, relação de equivalência 0,79 , ponto de ignição de 22 graus antes do PMS, velocidade de $1400 \mathrm{rpm}$ e uma pressão no coletor de admissão de 61,5 kPa. Os dados experimentais foram obtidos de Martínez(2016) [13]. Nota-se que os resultados obtidos na simulação representam satisfatoriamente o comportamento experimental.

Figura 6: Comparação dos dados de Matínez [13] com os resultados simulados.

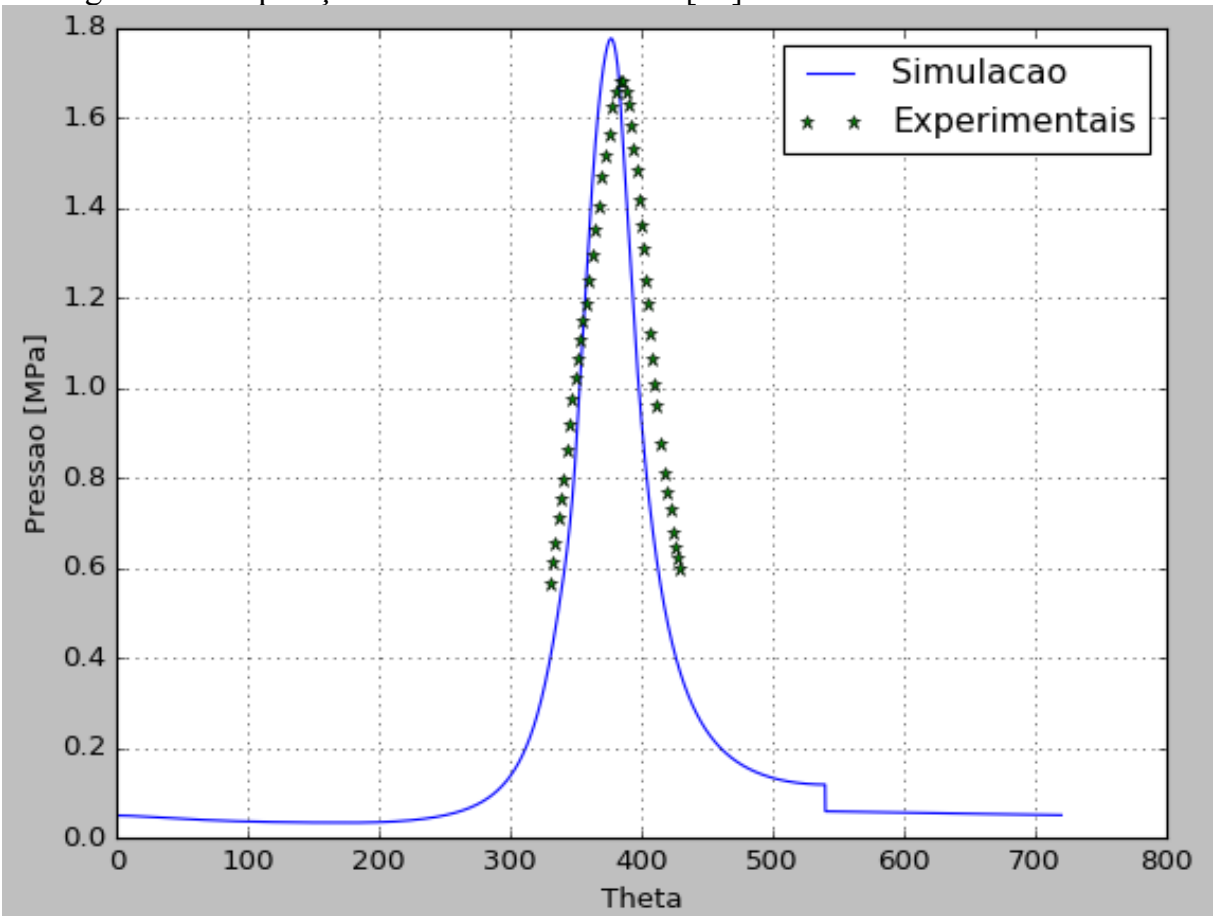

Fonte: Elaborado pelo próprio autor. 
Finalmente, a figura 7 mostra os resultados obtidos da simulação de um motor com taxa compressão de 9,5, diâmetro de $81 \mathrm{~mm}$, curso de $89 \mathrm{~mm}$, relação de equivalência 1, ponto de ignição de 20 graus antes do PMS, velocidade de $3500 \mathrm{rpm}$ e uma pressão no coletor de admissão de $50 \mathrm{kPa}$. Os dados experimentais foram obtidos de Yusri(2016) [14]. Neste caso, novamente, o comportamento e o pico de pressão são representados, de forma que os resultados podem ser considerados satisfatórios.

Figura 7: Comparação dos dados de Yusri [14] com os resultados simulados.

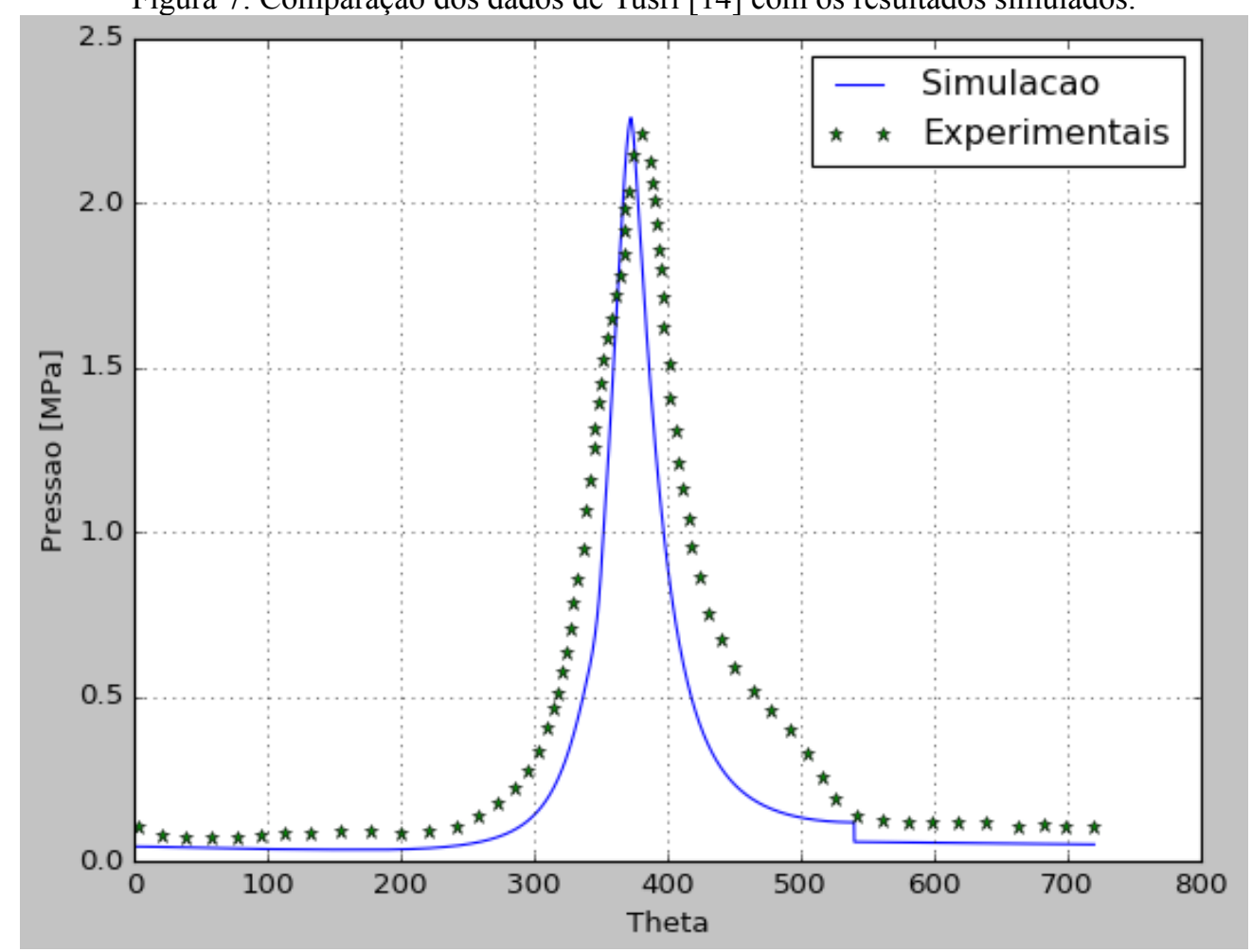

Fonte: Elaborado pelo próprio autor.

Grande parte dos desvios encontrados nos resultados das simulações em relação aos dados experimentais são devidos a causas já antes mencionadas, como, por exemplo, o modelo de trocas gasosas ser muito simplificado, desconsiderando efeitos de overlap e lift, e também pela ausência de modelos que considerem a influência da turbulência e do intervalo de tempo entre a ignição e o início da liberação de energia durante a combustão. Esses detalhes já estão sendo estudados e estarão presentes em versões futuras do código.

\section{CONCLUSÃO}

O modelo apresentado neste trabalho mostra ter um grande potencial para as análises de desempenho de motores de combustão interna. Por se tratar de uma formulação simplificada, os resultados são considerados satisfatórios, sendo que o objetivo inicial foi alcançado, uma vez que já é possível representar o comportamento de casos reais. Está previsto para trabalhos futuros a inserção de modelos mais robustos para questões como as trocas gasosas e atrito, além de módulos para a análise de parâmetros de desempenho e emissões. 
A linguagem de programação utilizada mostrou-se muito versátil e dinâmica para a implementação do código, além de ter uma disponibilidade muito grande de ferramentas. Porém, algumas funções apresentam velocidade de processamento inferior às outras linguagens concorrentes, de forma que com o avanço do modelo considera-se a possibilidade de utilizar códigos em Fortran para a realização de tarefas específicas.

\section{REFERÊNCIAS}

[1] CURTO-RISSO, P. L., et al. Quasi-Dimensional Simulation of Spark Ignition Engines. London: Springer, 2014.

[2] FENG, H., et al. Availability analysis of n-heptane/iso-octane blends during lowtemperature engine combustion using a single-zone combustion model. Energy Conversion and Management. China, v. 84, p. 613-622, 2014.

[3] HEYWOOD, J. B. Internal Combustion Engine Fundamentals. New York: McGraqHill Book Company, 1988.

[4] HVEZDA, J. Multi-Zone Models of Combustion and Heat Transfer Processes in SI Engines. Journal of Middle European Construction and Design of Cars. 10.2478/v10138012-0008-6, p. 14-22, 2012.

[5] MELO, T. C. C., Modelagem termodinâmica de um motor do ciclo Otto tipo flex-fuel, funcionando com gasolina, álcool e gás natural. Tese de M.Sc., COPPE/UFRJ, Rio de Janeiro, 2007.

[6] FERGUSON, C. R., KIRKPATRICK, A. T. Internal Combustion Engines Apllied Thermosciences. New York: John Wiley \& Sons, 2. ed., 2001.

[7] ANNAND, W. J. D., et al. Instantaneous Heat Transfer Rates to the Cylinder Head Surfece of a Small Compression-Ignition Engine. Proc. Inst. Mech. Engrs., v.185, p. 976-987, 19701971.

[8] WOSCHNI, G. Universally Applicable Equation for the Instantaneous Heat Transfer Coefficient in the Internal Combustion Engine. SAE paper, v. 76, n. 670931, 1967.

[9] PYTHON. Python is a programming language that lets you work quickly and integrate

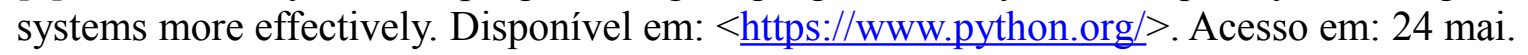
2017.

[10] MILLO, F. Esercitazione Volano. Turin: Politecnico di Torino. Publicação Interna.

[11] CHITRAGAR, P. R., et al. An experimental study on combustion and emission analysis of four cylinder 4-stroke gasoline engine using pure hydrogen and LPG at idle condition.

Energy Procedia. India, v. 90, p. 525-534, 2015.

[12] JI, C., et al. A quasi-dimensional model for combustion performance prediction of an SI hydrogen-enriched methanol engine. Hydrogen Energy., China, v. 41, p.17676-17686, 2016. 
[13] MARTÍNEZ-BOGGIO, S. D., et al. Simulation of cycle-to-cycle variations on spark ignition engines fueled with gasoline-hydrogen blends. Hydrogen Energy. Uruguai, v. 41, p. 9087-9099, 2016.

[14] YUSRI, I. M., et al. Experimental investigation of combustion, emissions and thermal balance of secondary butyl alcohol-gasoline blends in a spark ignition engine. Energy

Conversion and Management. Malaysia, v. 123, p. 1-14, 2016. 